\title{
ERRATUM
}

Celest. Mech., 58, 245-275

\section{HARRINGTON'S HAMILTONIAN IN THE STELLAR PROBLEM OF THREE BODIES; REDUCTIONS, RELATIVE EQUILIBRIA AND BIFURCATIONS}

FERRER S. and OSÁCAR C.

We have to point out that the Appendix is due to Dr. A. Deprit, which is the sole author of it.

Moreover, in the paper there are some misprints: Section 3.1,

In page 254, eleventh line, in Proposition 1 (part 3.1), it should read

$$
S_{R_{3}}^{2}=\left\{\left(\tilde{z}_{1}, \tilde{z}_{2}, \tilde{z}_{3}\right) \mid \tilde{z}_{1}^{2}+\tilde{z}_{2}^{2}+\tilde{z}_{3}^{2}=\frac{1}{4}\left(p_{2}+1\right)^{2}\right\}
$$

Section 4,

In page 256, there is a missing factor in the conditions verified by Poisson brackets of $\gamma$ coordinates, given after Equation (31) before the first paragraph. The correct expressions are

$$
\left\{\gamma_{1} ; \gamma_{2}\right\}=2 \frac{\eta_{1}}{L_{1}} \gamma_{3}, \quad\left\{\gamma_{2} ; \gamma_{3}\right\}=2 \frac{\eta_{1}}{L_{1}} \gamma_{1}, \quad\left\{\gamma_{3} ; \gamma_{1}\right\}=2 \frac{\eta_{1}}{L_{1}} \gamma_{2}
$$

In second line of page 257 , instead of 'according to (25)'+, it should read 'according to (31)'.

Section 4.1,

There is a misprint in the reduced function $P$ (p.257) which should read as:

$$
\begin{aligned}
P\left(\gamma_{1}, \gamma_{2}, \gamma_{3}\right)= & \left(5-3 \eta^{2}\right) \frac{3 \alpha^{2} \beta^{2}-\left(\alpha^{2}+4 \alpha \beta+\beta^{2}\right) \eta^{2}+3 \eta^{4}}{(\alpha-\beta)^{2} \eta^{2}} \\
& +15 \frac{1-\eta^{2}}{\eta^{2}} \frac{\alpha^{2}-\eta^{2}}{\omega^{2}-\eta^{2}} \frac{\gamma_{1}^{2}-\gamma_{2}^{2}}{(\alpha-\beta)^{2}}
\end{aligned}
$$

Equations of motion (32) should be changed by

$$
\frac{d \gamma}{d \tau}=\{\gamma ; P\}=2 \frac{\eta_{1}}{L_{1}} \nabla_{\gamma} P \times \gamma
$$

The corresponding equations (33) to (38) should read now multiplying their right hand members by the factor $2 \eta_{1} / L_{1}$. This factor does not affect results in the rest of the paper. 